TRANSACTIONS OF THE

AMERICAN MATHEMATICAL SOCIETY

Volume 348, Number 1, January 1996

\title{
THE SPACE OF INVARIANT FUNCTIONS ON A FINITE LIE ALGEBRA
}

\author{
G. I. LEHRER
}

\begin{abstract}
We show that the operations of Fourier transform and duality on the space of adjoint-invariant functions on a finite Lie algebra commute with each other. This result is applied to give formulae for the Fourier transform of a "Brauer function" - i.e. one whose value at $X$ depends only on the semisimple part $X_{s}$ of $X$ and for the dual of the convolution of any function with the Steinberg function. Geometric applications include the evaluation of the characters of the Springer representations of Weyl groups and the study of the equivariant cohomology of local systems on $G / T$, where $T$ is a maximal torus of the underlying reductive group $G$.
\end{abstract}

\section{INTRODUCTION AND NOTATION}

Let $G$ be a connected reductive group defined over the finite field $\mathbb{F}_{q}$ and let $F: G \longrightarrow G$ be the Frobenius endomorphism associated with the $\mathbb{F}_{q}$-structure on $G$. Let $\mathfrak{G}=$ Lie $G$ be the Lie algebra of $G$. Then there is an induced action of $F$ on $\mathfrak{G}$. The group $G$ acts on $\mathfrak{G}$ by means of the adjoint representation and we denote by $A d(g)$ the linear transformation of $\mathfrak{G}$ corresponding to $g \in G$. For any variety $V$ upon which $F$ acts, if $S$ is an $F$-stable subvariety of $V$, we denote by $S^{F}$ the set of $F$-fixed (or $\mathbb{F}_{q}$-rational) points of $S$.

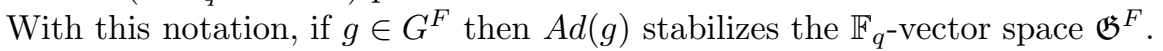

(1.1) Definition. The space $\mathcal{C}\left(\mathfrak{G}^{F}\right)$ of invariant functions on $\mathfrak{G}^{F}$ is the space of functions $f: \mathfrak{G}^{F} \longrightarrow \mathbb{C}$ which are invariant under the action of $G^{F}$, i.e. such that $f(A d(g) X)=f(X)$ for all $g \in G^{F}$ and $X \in \mathfrak{G}^{F}$.

The principal source of interest in $\mathcal{C}\left(\mathfrak{G}^{F}\right)$ has been its relevance for the character theory of the finite groups $G^{F}$. The work of Deligne and Lusztig [DL] showed that a key to understanding this theory lay in the study of the values of characters on unipotent elements. Springer [Sp3] has shown that there is a $G$-equivariant bijection: $G_{\text {uni }} \longrightarrow \mathfrak{G}_{\text {nil }}$ from the unipotent variety of $G$ ( $G$ acting by conjugation) to the nilpotent variety of $\mathfrak{G}$ (given certain restrictions on the characteristic). This led to the study of nilpotently supported functions in $\mathcal{C}\left(\mathfrak{G}^{F}\right)$.

The main purpose of the present work is to prove that the Fourier transform commutes with the duality map (Theorem 4.6 below) in the context of a general structure theory for $\mathcal{C}\left(\mathfrak{G}^{F}\right)$, and to give applications of this theorem and other results to the theory to Green functions and other "character formulae" in $\mathcal{C}\left(\mathfrak{G}^{F}\right)$. One of the applications $((7.13))$ gives the trace of $F$ on $H_{c}^{*}\left(\mathcal{T}_{X}, \mathcal{S}_{\epsilon}\right)$ where $\mathcal{S}_{\epsilon}$ is a

Received by the editors February 15, 1994.

1991 Mathematics Subject Classification. Primary 20G40, 20G05; Secondary 22E60, 11 T24. 
certain $\ell$-adic local system (see (7.11)) on the subvariety $\mathcal{T}_{X}$ of the variety $\mathcal{T}$ of maximal tori of $G$. This suggests a definition of a "Steinberg character" for real Lie groups and also suggests some results on Euler characteristics of certain subvarieties of the variety of maximal tori of a real Lie group.

The key to these results is an operation $\mathcal{F}$ (the "Fourier transform") on $\mathcal{C}\left(\mathfrak{G}^{F}\right)$ which has no counterpart in the group case. The definition of $\mathcal{F}$ imposes mild restrictions (cf. [Sp1]) on the characteristic because of the

(1.2) Assumption. There is a non-singular bilinear form $\langle\rangle:, \mathfrak{G} \times \mathfrak{G} \longrightarrow \overline{\mathbb{F}}_{q}$ which satisfies

(i) $\langle$,$\rangle is defined over \mathbb{F}_{q}$ and

(ii) $\langle$,$\rangle is A d G$-invariant, i.e. for $g \in G,\langle\operatorname{Ad}(g) X, A d(g) Y\rangle=\langle X, Y\rangle$ for all $X, Y \in \mathfrak{G}$.

This paper is organised as follows. In $\S 2$ we discuss the elementary properties of $\mathcal{C}\left(\mathfrak{G}^{F}\right)$, including the inner product, restriction and induction maps and convolution. In $\S 3$ we discuss the duality operation $\mathcal{D}: \mathcal{C}\left(\mathfrak{G}^{F}\right) \longrightarrow \mathcal{C}\left(\mathfrak{G}^{F}\right)$ introduced by Kawanaka $([\mathrm{K}])$ in the context of $\mathcal{C}\left(\mathfrak{G}^{F}\right)$ and by Lusztig and Curtis-Alvis $[\mathrm{C}]$ in the group context. The results depend on a Lie algebra analogue of a "SchurZassenhaus"- type conjugacy result (cf. (3.7) below) and involve a discussion of the space of "Brauer functions" on $\mathfrak{G}^{F}$, including its relationship with the Steinberg function on $\mathfrak{G}^{F}$.

Section 4 deals with the Fourier transform $\mathcal{F}: \mathcal{C}\left(\mathfrak{G}^{F}\right) \longrightarrow \mathcal{C}\left(\mathfrak{G}^{F}\right)$. After a general discussion of its properties we prove

Theorem 4.6. Let $\mathcal{D}$ be the duality operation on $\mathcal{C}\left(\mathfrak{G}^{F}\right)$ and let $\mathcal{F}$ be the Fourier transform on $\mathcal{C}\left(\mathfrak{G}^{F}\right)$. Then $\mathcal{D} \mathcal{F}=\mathcal{F} \mathcal{D}$.

In $\S 5$ we give applications of the foregoing results to the computation of certain character values. Among other things we recover Springer's result that $\mathcal{F} S t_{\mathfrak{G}}=$ $q^{r / 2} \eta_{0}$ (where $\eta_{0}$ is the characteristic function of the nilpotent set of $\mathfrak{G}^{F}$ ) and give a general formula for the Fourier transform of a "Brauer function" (one which satisfies $f(X)=f\left(X_{s}\right)$ for $\left.X \in \mathfrak{G}^{F}\right)$ on $\mathfrak{G}^{F}$. In $\S 6$, we give a "closed formula" for the Green function $\tilde{Q}_{w}(X)=\sum_{i} \operatorname{tr}\left(w F, H^{2 i}\left(\mathcal{B}_{X}, \bar{Q}_{\ell}\right)\right)$ as a rational multiple of $\xi_{S_{w}} * S t_{\mathfrak{G}}$, where $\xi_{S_{w}}$ is the characteristic function of the orbit of the regular semisimple element $S_{w} \in \mathfrak{G}^{F}$ and $*$ is convolution in $\mathcal{C}\left(\mathfrak{G}^{F}\right)$.

Finally in $\S 7$, we interpret some of our formulae in terms of the trace of Frobenius on $H_{c}^{*}\left(V, \mathcal{S}_{\epsilon}\right)$ where $V$ is a subvariety of the variety $\mathcal{T}$ of maximal tori of $G$ and $\mathcal{S}_{\epsilon}$ is a certain irreducible local system on $V$. The results in this section have obvious analogues for real Lie groups and we shall address that situation elsewhere.

\section{THE SPACE $\mathcal{C}\left(\mathfrak{G}^{F}\right)$}

In this section we review some of the elementary properties of $\mathcal{C}\left(\mathfrak{G}^{F}\right)$.

(2.1) Definition. For $f, g \in \mathcal{C}\left(\mathfrak{G}^{F}\right)$ define the positive definite non-singular Hermitian form $()=,(,)_{\mathfrak{G}^{F}}$ by

$$
(f, g)=\left|G^{F}\right|^{-1} \sum_{X \in \mathfrak{G}^{F}} f(X) \overline{g(X)} .
$$


Let $H$ be a closed F-stable (i.e. rational) subgroup of $G$. Even if $H$ is not connected and reductive, we may speak of the inner product space $\mathcal{C}\left(\mathfrak{H}^{F}\right)$ (where $\mathfrak{H}$ $=$ Lie $(H)$ ). Since $\mathfrak{H}$ is a subalgebra of $\mathfrak{G}$ (identified as derivations which annihilate the ideal of $H$ ) we have the maps

$$
\operatorname{Res}_{\mathfrak{H}}^{\mathfrak{H}}: \mathcal{C}\left(\mathfrak{G}^{F}\right) \longrightarrow \mathcal{C}\left(\mathfrak{H}^{F}\right)
$$

given by restriction of functions and

$$
\operatorname{Ind}_{\mathfrak{H}}^{\mathfrak{G}}: \mathcal{C}\left(\mathfrak{H}^{F}\right) \longrightarrow \mathcal{C}\left(\mathfrak{G}^{F}\right)
$$

defined by

$$
\begin{array}{rl}
\operatorname{Ind}_{\mathfrak{H}}^{\mathfrak{G}} f(X)=\left|H^{F}\right|^{-1} \sum_{\substack{g \in G^{F} \\
A d(g) X \in \mathfrak{H}^{F}}} & f(A d(g) X) \\
& \left(\text { for } f \in \mathcal{C}\left(\mathfrak{H}^{F}\right), X \in \mathfrak{G}^{F}\right) .
\end{array}
$$

A simple computation shows that Res and Ind are adjoint with respect to the inner products on $\mathcal{C}\left(\mathfrak{G}^{F}\right)$ and $\mathcal{C}\left(\mathfrak{H}^{F}\right)$ respectively, i.e.

$$
\text { For } f \in \mathcal{C}\left(\mathfrak{G}^{F}\right), g \in \mathcal{C}\left(\mathfrak{H}^{F}\right) \text { we have }
$$

$$
\left(\operatorname{Res}_{\mathfrak{H}}^{\mathfrak{G}} f, g\right)_{\mathfrak{H}^{F}}=\left(f, \operatorname{Ind}_{\mathfrak{H}}^{\mathfrak{G}} g\right)_{\mathfrak{G} F}
$$

In addition to pointwise multiplication of functions in $\mathcal{C}\left(\mathfrak{G}^{F}\right)$, which will be denoted by juxtaposition $\left(f g(X)=f(X) g(X)\right.$ for $\left.f, g \in \mathcal{C}\left(\mathfrak{G}^{F}\right), X \in \mathfrak{G}^{F}\right)$, we have convolution defined by

$$
\begin{aligned}
& \text { For } f, g \in \mathcal{C}\left(\mathfrak{G}^{F}\right) \text { define the convolution product } f * g \text { by } \\
& \qquad f * g(X)=\left|\mathfrak{G}^{F}\right|^{-1 / 2} \sum_{Y \in \mathfrak{G}^{F}} f(X-Y) g(Y) \\
& \qquad\left(f, g \in \mathcal{C}\left(\mathfrak{G}^{F}\right), X \in \mathfrak{G}^{F}\right) .
\end{aligned}
$$

The following properties are easily verified

\section{(2.5) Lemma.}

(i) Convolution is an associative and commutative product on $\mathcal{C}\left(\mathfrak{G}^{F}\right)$, with corresponding identity element $\left|\mathfrak{G}^{F}\right|^{1 / 2} \xi_{0}$, where $\xi_{0}(X)=1$ if $X=0$ and $\xi_{0}(X)=0$ otherwise.

(ii) We have, for $f, g, h \in \mathcal{C}\left(\mathfrak{G}^{F}\right)$,

$$
(f * g, h)=\left(f, g^{*} * h\right)_{\mathfrak{G}^{F}}
$$

where $g^{*}(X)=\overline{g(-X))}$.

Let $H, K$ be two closed F-stable subgroups of $G$. We then have the following "Mackey formula" (see [Sp1]) 
(2.6) Lemma (Springer). With the above notation, write $\mathfrak{H}=$ LieH, $\mathfrak{K}=$ LieK, and assume that for any element $g \in G^{F}$, Lie $\left(K \cap^{g} H\right)=\mathfrak{K} \cap A d(g) \mathfrak{H}$.

Then for $f \in \mathcal{C}\left(\mathfrak{H}^{F}\right)$, we have

$$
\operatorname{Res}_{\mathfrak{K}}^{\mathfrak{G}} \circ \operatorname{In} d_{\mathfrak{H}}^{\mathfrak{G}} f=\sum_{g \in K \backslash G / H} \operatorname{In} d_{\mathfrak{K} \cap A d(g) \mathfrak{H}}^{\mathfrak{K}} \circ \operatorname{Res}_{\mathfrak{K} \cap A d(g) \mathfrak{H}}^{\operatorname{Ad}(g) \mathfrak{H}}\left(f \circ A d(g)^{-1}\right) .
$$

Most properties enjoyed by group characters also apply in $\mathcal{C}\left(\mathfrak{G}^{F}\right)$. We mention one which we use below.

(2.7) Lemma. Let $H$ be a rational closed subgroup of $G, \mathfrak{H}=$ Lie $H$. Let $f \in$ $\mathcal{C}\left(\mathfrak{G}^{F}\right), g \in \mathcal{C}\left(\mathfrak{H}^{F}\right)$. Then $\operatorname{In} d_{\mathfrak{H}}^{\mathfrak{G}}\left(\operatorname{Res}_{\mathfrak{H}}^{\mathfrak{G}} f \cdot g\right)=f \operatorname{Ind} d_{\mathfrak{H}}^{\mathfrak{G}}(g)$.

\section{HARISH-CHANDRA INDUCTION AND DUALITY}

Let $P=L U$ be an F-stable Levi decomposition of the F-stable parabolic subgroup $P$ of $G$. Let $\mathfrak{P}=\mathfrak{L}+\mathfrak{U}$ be the corresponding Levi decomposition of $\mathfrak{P}=\operatorname{Lie}(P)$.

(3.1) Definition.

(i) The truncation map $\tau_{\mathfrak{L} \subset \mathfrak{P}}^{\mathfrak{G}}: \mathcal{C}\left(\mathfrak{G}^{F}\right) \longrightarrow \mathcal{C}\left(\mathfrak{L}^{F}\right)$ is defined by

$$
\begin{array}{rl}
\tau_{\mathfrak{L} \subset \mathfrak{P}}^{\mathfrak{G}} f(X)=\left|\mathfrak{U}^{F}\right|^{-1} \sum_{Y \in \mathfrak{U}^{F}} & f(X+Y) \\
& \left(f \in \mathcal{C}\left(\mathfrak{G}^{F}\right), X \in \mathfrak{L}^{F}\right) .
\end{array}
$$

(ii) The Harish-Chandra induction map $\rho_{\mathfrak{L} \subset \mathfrak{G}}^{\mathfrak{G}}: \mathcal{C}\left(\mathfrak{L}^{F}\right) \longrightarrow \mathcal{C}\left(\mathfrak{G}^{F}\right)$ is defined by $\rho_{\mathfrak{L} \subset \mathfrak{P}}^{\mathfrak{G}} g=\operatorname{Ind}_{\mathfrak{P}}^{\mathfrak{G}} \tilde{g}$, where $\tilde{g} \in \mathcal{C}\left(\mathfrak{P}^{F}\right)$ is given by $\tilde{g}(X+Y)=g(X)(X \in$ $\left.\mathfrak{L}^{F}, Y \in \mathfrak{U}^{F}, g \in \mathcal{C}\left(\mathfrak{L}^{F}\right)\right)$

(3.2) Lemma. The maps $\tau_{\mathfrak{L} \subset \mathfrak{P}}^{\mathfrak{G}}$ and $\rho_{\mathfrak{L} \subset \mathfrak{P}}^{\mathfrak{G}}$ are adjoint with respect to the inner products $(,)_{\mathfrak{G}^{F}}$ and $(,)_{\mathfrak{L}^{F}}$ on $\mathcal{C}\left(\mathfrak{G}^{F}\right)$ and $\mathcal{C}\left(\mathfrak{L}^{F}\right)$ respectively.

Proof. Let $f \in \mathcal{C}\left(\mathfrak{G}^{F}\right)$ and $g \in \mathcal{C}\left(\mathfrak{L}^{F}\right)$.

Then

$$
\begin{aligned}
\left(f, \rho_{\mathfrak{L} \subset \mathfrak{P}}^{\mathfrak{G} g}\right)_{\mathfrak{G}^{F}} & =\left|G^{F}\right|^{-1} \sum_{X \in \mathfrak{G}^{F}} f(X) \operatorname{ind}_{\mathfrak{P}}^{\mathfrak{G} \tilde{g}(X)} \\
& =\left|G^{F}\right|^{-1} \sum_{x \in \mathfrak{G}^{F}} f(X) \sum_{\substack{x \in G^{F} \\
A d x X \in \mathfrak{P}^{F}}} \overline{\tilde{g}(A d x X)} .
\end{aligned}
$$

Reversing the order of summation, the latter becomes

$$
\left|G^{F}\right|^{-1}\left|P^{F}\right|^{-1} \sum_{x \in G^{F}} \sum_{X \in A d x^{-1} \mathfrak{P}^{F}} f(A d x X) \overline{\tilde{g}(A d x X)} .
$$

The inner sum is clearly equal to $\left|P^{F}\right|\left(\tau_{\mathfrak{L} \subset \mathfrak{P}}^{\mathfrak{G}} f, g\right)_{\mathfrak{L}^{F}}$ for each $x \in\left|G^{F}\right|$, which proves the result. 
(3.3) Proposition. The maps $\tau_{\mathfrak{L} \subset \mathfrak{P}}^{\mathfrak{G}}$ and $\rho_{\mathfrak{L} \subset \mathfrak{P}}^{\mathfrak{G}}$ are independent of $\mathfrak{P}$; i.e. given $L$ and two F-stable parabolic subgroups $P_{1} \supset L$ and $P_{2} \supset L$, we have $\tau_{\mathfrak{L} \subset \mathfrak{P}_{1}}^{\mathfrak{G}}=\tau_{\mathfrak{L} \subset \mathfrak{P}_{2}}^{\mathfrak{G}}$ where $\mathfrak{P}_{i}=\operatorname{Lie}\left(P_{i}\right)(i=1,2)$ and similarly for $\rho$.

Proof. By (3.2) it is clearly sufficient to prove this for $\rho$, since by the non-degenerate nature of $(,)_{\mathfrak{L}^{F}}$, if $\rho_{\mathfrak{L}^{\prime} \subset \mathfrak{P}_{1}}^{\mathfrak{G}}=\rho_{\mathfrak{L} \subset \mathfrak{P}_{2}}^{\mathfrak{G}}$ we have, for any $f \in \mathcal{C}\left(\mathfrak{G}^{F}\right)$ and $g \in$ $\mathcal{C}\left(\mathfrak{L}^{F}\right),\left(\tau_{\mathfrak{L}^{\prime} \subset \mathfrak{P}_{1}}^{\mathfrak{G}} f, g\right)_{\mathfrak{L}^{F}}=\left(f, \rho_{\mathfrak{L} \subset \mathfrak{P}_{1}}^{\mathfrak{G}} g\right)_{\mathfrak{G}^{F}}=\left(f, \rho_{\mathfrak{L} \subset \mathfrak{P}_{2}}^{\mathfrak{G}} g\right)_{\mathfrak{G}^{F}}=\left(\tau_{\mathfrak{L} \subset \mathfrak{P}_{2}}^{\mathfrak{G}} f, g\right)_{\mathfrak{L}^{F}}$. Thus $\tau_{\mathfrak{L} \subset \mathfrak{P}_{1}}^{\mathfrak{G}}=\tau_{\mathfrak{L} \subset \mathfrak{P}_{2}}^{\mathfrak{G}}$.

To prove the result for $\rho$ one can proceed exactly as in the proof of the corresponding result for the group case (see [DM], Proposition 6.1, page 57) since the argument used there utilises only the Mackey formula which is available here $((2.6)$ above). This is used to show that for $f \in \mathcal{C}\left(\mathfrak{G}^{F}\right),\left(\rho_{\mathfrak{L} \subset \mathfrak{P}_{1}}^{\mathfrak{G}} f, \rho_{\mathfrak{L} \subset \mathfrak{P}_{2}}^{\mathfrak{G}} f\right)=\left(\rho_{\mathfrak{L} \subset \mathfrak{P}_{1}}^{\mathfrak{G}} f, \rho_{\mathfrak{L} \subset \mathfrak{P}_{1}}^{\mathfrak{G}} f\right)=$ $\left(\rho_{\mathfrak{L} \subset \mathfrak{P}_{2}}^{\mathfrak{G}} f, \rho_{\mathfrak{L} \subset \mathfrak{P}_{2}}^{\mathfrak{G}} f\right)$ which by the non-degenerate nature of $(,)_{\mathfrak{G}^{F}}$ gives the result.

Alternatively, the argument of [HL] may be adapted to the present situation.

In view of (3.3), we shall henceforth write $\tau_{\mathfrak{L}}^{\mathfrak{G}}$ for $\tau_{\mathfrak{L} \subset \mathfrak{P}}^{\mathfrak{G}}$ etc.

Next, we define (following Kawanaka $[\mathrm{K}]$ in the Lie algebra case and Lusztig, Curtis and Alvis in the group case) the "duality map" $\mathcal{D}: \mathcal{C}\left(\mathfrak{G}^{F}\right) \longrightarrow \mathcal{C}\left(\mathfrak{G}^{F}\right)$. Let $T_{0}$ be an F-stable maximal torus of $G$ which is contained in an F-stable Borel subgroup $B$. Then any parabolic subgroup $P \supset B$ contains a unique Levi subgroup $L_{P} \supset T_{0}$.

(3.4) Definition. Let $T_{0} \subset B$ be as above. For $f \in \mathcal{C}\left(\mathfrak{G}^{F}\right)$, write

$$
\mathcal{D} f=\sum_{\substack{P \supset B \\ F P=P}}(-1)^{n(P)} \rho_{\mathfrak{L}_{P}}^{\mathfrak{G}} \circ \tau_{\mathfrak{L}_{P}}^{\mathfrak{G}} f
$$

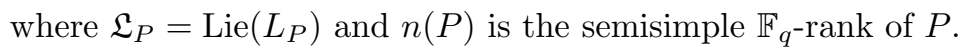

(3.5) Proposition (Kawanaka $[K, \S 4]$ ).

(i) The linear map $\mathcal{D}$ is an isometry with respect to the inner product $(,)_{\mathfrak{G}}^{F}$.

(ii) $\mathcal{D}$ is an involution, i.e. $\mathcal{D}^{2}=i d_{\mathcal{C}\left(\mathfrak{G}^{F}\right)}$.

(3.6) Proposition (Springer [Sp1]). Denote by St $t_{\mathfrak{G}}$ the "Steinberg function" on $\mathfrak{G}^{F}$ defined by $S t_{\mathfrak{G}}=\mathcal{D} 1_{\mathfrak{G}}$, where $1_{\mathfrak{G}}(X)=1$ for all $X \in \mathfrak{G}^{F}$. Then $S t_{\mathfrak{G}}$ has values given by

$$
S t_{\mathfrak{G}}(X)= \begin{cases}0 & \text { unless } X \text { is semisimple } \\ \epsilon(X)\left|C_{G}(X)^{0 F}\right|_{p} & \text { if } X \text { is semisimple }\end{cases}
$$

where $n_{p}$ denotes the $p$-part ( $p$ a prime) of the integer $n, p$ is the characteristic of $\mathbb{F}_{q}$ and $\epsilon(X)=(-1)^{\left(r(G)-r\left(C_{G}(X)^{\circ}\right)\right)}\left(r(H)\right.$ denotes the $\mathbb{F}_{q}$-rank of the $\mathbb{F}_{q}$-group $H$.)

This was proved by Springer [loc. cit.] as an application of the results of [CLT]. A key property of the map $\mathcal{D}$ will be a consequence of the following "SchurZassenhaus"- type result for Lie algebras, which is the Lie algebra analogue of a known result for groups (cf. [L2] or [MS]).

(3.7) Proposition. Let $P=L U$ be a Levi decomposition of the parabolic subgroup $P$ of the connected reductive group $G$. Let $\mathfrak{P}=\mathfrak{L} \oplus \mathfrak{U}$ be the corresponding decom- 
position of its Lie algebra and let $X \in \mathfrak{L}, N \in \mathfrak{U}$. Then $(X+N)_{s}=\operatorname{Adv}\left(X_{s}\right)$ for some $v \in U$.

Proof. By Borel [B], (14.16) there is a Borel subgroup $B_{L}=T V$ of $L$ such that $X_{s} \in \operatorname{Lie}(T)$ and $X_{n} \in \operatorname{Lie}(V)$. Then $B=B_{L} U=T$.VU is a Borel subgroup of $G$ and its Lie algebra splits as $\operatorname{Lie}(B)=\operatorname{Lie}(T) \oplus \operatorname{Lie}(V) \oplus \mathfrak{U}$.

Now $(X+N)_{s}$ is a semisimple element of $\operatorname{Lie}(B)$, whence $(X+N)_{s}=\operatorname{Adv}(Y)$, for some $Y \in \operatorname{Lie}(T)$ and $v \in V U$. But the map $\mu_{v}: T \longrightarrow V U$ defined by $\mu_{v}(t)=t^{-1} v t v^{-1}$ has derivative $\left(-i d_{L i e(T)}+A d v\right): \operatorname{Lie}(T) \longrightarrow \operatorname{Lie}(V U)$. Hence $\operatorname{Adv}(Y)-Y \in \operatorname{Lie}(V U)$, whence $X+N=(X+N)_{s}+(X+N)_{n} \in Y+\operatorname{Lie}(V U)$. However we also have $X+N=X_{s}+X_{n}+N \in X_{s}+\operatorname{Lie}(V U)$, so that $Y=X_{s}$. Now in the above argument, $L$ may be replaced by $C_{L}\left(X_{s}\right)$. This shows that the element $v$ may be taken in $U$.

(3.8) Definition. Define two subspaces of $\mathcal{C}\left(\mathfrak{G}^{F}\right)$ as follows:

$$
\begin{aligned}
& \mathcal{C}\left(\mathfrak{G}^{F}\right)_{s s}=\left\{f \in \mathcal{C}\left(\mathfrak{G}^{F}\right) \mid f(X)=0 \text { unless } X=X_{s}\right\} . \\
& \mathcal{B}\left(\mathfrak{G}^{F}\right)=\left\{f \in \mathcal{C}\left(\mathfrak{G}^{F}\right) \mid f(X)=f\left(X_{s}\right) \text { for } X \in \mathfrak{G}^{F}\right\} .
\end{aligned}
$$

(3.9) Corollary. Let $G$ be such that $C_{G}(X)$ is connected if $X$ is semisimple. Let $P=L U$ be an F-stable Levi decomposition of the parabolic subgroup $P$ of $G$, and suppose $b \in \mathcal{B}\left(\mathfrak{G}^{F}\right)$. If $\mathfrak{L}=$ Lie $L$ and $X \in \mathfrak{L}^{F}$, then for any element $f \in \mathcal{C}\left(\mathfrak{G}^{F}\right)$, we have $\tau_{\mathfrak{L}}^{\mathfrak{G}}(b f)(X)=b(X) \tau_{\mathfrak{L}}^{\mathfrak{G}} f(X)$

Proof. We have

$$
\begin{aligned}
\tau_{\mathfrak{L}}^{\mathfrak{G}}(b f)(X) & =\left|\mathfrak{U}^{F}\right|^{-1} \sum_{Y \in \mathfrak{U}^{F}} b f(X+Y) \\
& =\left|\mathfrak{U}^{F}\right|^{-1} \sum_{Y \in \mathfrak{U}^{F}} b\left((X+Y)_{s}\right) f(X+Y)
\end{aligned}
$$

since $b \in \mathcal{B}\left(\mathfrak{G}^{F}\right)$.

But by Theorem (3.7), if $Y \in \mathfrak{U}^{F}$, then $(X+Y)_{s}$ and $X_{s}$ are in the same $A d G$ orbit. Moreover since $C_{G}\left(X_{s}\right)$ is connected, this implies that they are in the same $A d G^{F}$ orbit on $\mathfrak{G}^{F}$. Thus $b\left((X+Y)_{s}\right)=b\left(X_{s}\right)=b(X)$, and the result follows.

(3.10) Theorem. Let $f \in \mathcal{C}\left(\mathfrak{G}^{F}\right)$ and $b \in \mathcal{B}\left(\mathfrak{G}^{F}\right)$ as above. Then

$$
\mathcal{D}(b f)=b \mathcal{D} f .
$$

Proof. We have, in the notation of (3.4),

$$
\begin{aligned}
\mathcal{D}(b f) & =\sum_{\substack{P \supset B \\
F P=P}}(-1)^{n(P)} \rho_{\mathfrak{L}}^{\mathfrak{G}} \circ \tau_{\mathfrak{L}}^{\mathfrak{G}}(b f) \\
& =\sum_{\substack{P \supset B \\
F P=P}}(-1)^{n(P)} \rho_{\mathfrak{L}}^{\mathfrak{G}}\left(\operatorname{Res}_{\mathfrak{L}}^{\mathfrak{G}} b \cdot \tau_{\mathfrak{L}}^{\mathcal{F}} f\right) \quad(\text { by } 3.9) .
\end{aligned}
$$


But (in the notation of (3.1) (ii)) if $X \in \mathfrak{L}^{F}$ and $Y \in \mathfrak{U}^{F}$,

$$
\begin{aligned}
\operatorname{Res}_{\mathfrak{L}}^{\widetilde{\mathfrak{G}} b \cdot \tau_{\mathfrak{L}}^{\mathfrak{G}} f(X+Y)} & =b(X) \widetilde{\tau_{\mathfrak{L}}^{\mathfrak{G}} f}(X+Y) \\
& =b(X s) \widetilde{\tau_{\mathfrak{L}}^{\mathfrak{G}} f}(X+Y) \\
& =b\left((X+Y)_{s}\right) \widetilde{\tau_{\mathfrak{L}}^{\mathfrak{G}}} f(X+Y) \\
& =b(X+Y) \widetilde{\tau_{\mathfrak{L}}^{\mathfrak{G}} f}(X+Y) .
\end{aligned}
$$

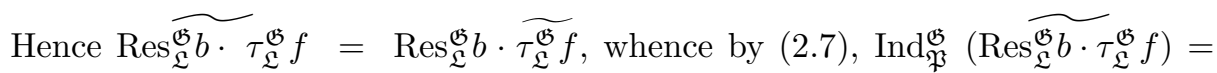
$b \cdot \operatorname{Ind}_{\mathfrak{P}}^{\mathfrak{G}}\left(\widetilde{\tau_{\mathfrak{L}}^{\mathfrak{G}} f}\right)$.

The result now follows by substituting this latter result into the right hand side of (3.10.1).

(3.11) Corollary.

(i) Let $b \in \mathcal{B}\left(\mathfrak{G}^{F}\right)$. Then $\mathcal{D} b=b \cdot S t_{\mathfrak{G}}$.

(ii) The map $\mathcal{D}$ induces an involutive isometric isomorphism between the spaces $\mathcal{B}\left(\mathfrak{G}^{F}\right)$ and $\mathcal{C}\left(\mathfrak{G}^{F}\right)_{s s}$.

Proof. For (i), take $g=1_{\mathfrak{G}}$ in (3.10). The statement (ii) is clear from (i) and (3.6).

(3.12) Proposition. Let $b \in \mathcal{B}\left(\mathfrak{G}^{F}\right)$ and $f_{1}, f_{2} \in \mathcal{C}\left(\mathfrak{G}^{F}\right)$. Then

$$
\left(f_{1} f_{2}, b\right)=\left(\mathcal{D} f_{1} \cdot \mathcal{D} f_{2}, b\right) .
$$

Proof. We have

$$
\begin{aligned}
\left(f_{1} f_{2}, b\right) & =\left(f_{1}, \bar{f}_{2} b\right)=\left(\mathcal{D} f_{1}, \mathcal{D}\left(\bar{f}_{2} b\right)\right) \\
& =\left(\mathcal{D} f_{1}, \mathcal{D}\left(\bar{f}_{2}\right) b\right) \text { by }(3.10) \\
& =\left(\mathcal{D} f_{1} \cdot \mathcal{D} f_{2}, b\right) \text { since }\left(\mathcal{D} \bar{f}_{2}\right)=\overline{\left(\mathcal{D} f_{2}\right)} .
\end{aligned}
$$

(3.13) Proposition. Assume that for $X \in \mathfrak{G}_{s s}^{F}, C_{G}(X)$ is connected. Let $f_{1}$ and $f_{2} \in \mathcal{C}\left(\mathfrak{G}^{F}\right)$ and let $S$ be a semisimple element of $\mathfrak{G}^{F}$. Then

$$
\sum_{N \in \mathfrak{L}_{n i l}^{F}} f_{1}(S+N) f_{2}(S+N)=\sum_{N \in \mathfrak{L}_{n i l}^{F}} \mathcal{D} f_{1}(S+N) \mathcal{D} f_{2}(S+N)
$$

where $\mathfrak{L}=C_{\mathfrak{G}}(S)\left(=\operatorname{Lie}(L), L=C_{G}(X)^{0}\right)$ and $\mathfrak{L}_{\text {nil }}$ is the nilpotent set in $\mathfrak{L}$.

Proof. Define $\eta_{S} \in \mathcal{B}\left(\mathfrak{G}^{F}\right)$ (cf. (5.1) below) by $\eta_{S}(Y)=1$ if $Y_{s}$ is the $G^{F}$-orbit $\mathcal{O}_{S}$ of $S$ and $\eta_{S}(Y)=0$ otherwise. Note that $A=\left\{Y \in \mathfrak{G}^{F} \mid Y_{s} \in \mathcal{O}_{S}\right\}$ is a disjoint union: $A=\coprod_{X \in \mathcal{O}_{S}} A_{X}$, where $A_{X}=\left\{Y \in A \mid Y_{s}=X\right\}$.

Moreover each set $A_{X}$ is $G^{F}$-conjugate to $A_{S}=\left\{S+N \mid N \in \mathfrak{L}_{\text {nil }}^{F}\right\}$.

Now let $f \in \mathcal{C}\left(\mathfrak{G}^{F}\right)$. Then

$$
\left(\eta_{S}, f\right)=\left|G^{F}\right|^{-1} \sum_{Y \in A} f(Y)=\left|G^{F}\right|^{-1} \sum_{X \in \mathcal{O}_{S}} \sum_{Y \in A_{X}} f(Y) .
$$


Since $f$ is $A d G^{F}$-invariant and the sets $A_{X}$ are $A d G^{F}$-conjugate, each inner $\operatorname{sum} \sum_{Y \in A_{X}} f(Y)$ is equal to $\sum_{Y \in A_{S}} f(Y)$. But $\left|\mathcal{O}_{S}\right|=\left|G^{F}\right|\left|L^{F}\right|^{-1}$, whence from (3.13.1) we obtain

$$
\left(\eta_{S}, f\right)=\left|L^{F}\right|^{-1} \sum_{N \in \mathfrak{L}_{n i l}^{F}} f(S+N)
$$

Next observe that since $\eta_{S} \in \mathcal{B}\left(\mathfrak{G}^{F}\right)$, we have $\left(\eta_{S}, f_{1} f_{2}\right)=\left(\eta_{S}, \mathcal{D} f_{1} \cdot \mathcal{D} f_{2}\right)$ by (3.12). Putting $f=f_{1} f_{2}$ and $f=\mathcal{D} f_{1} \mathcal{D} f_{2}$ respectively in (3.13.2) gives the stated equation.

Corollary. Let $f \in \mathcal{C}\left(\mathfrak{G}^{F}\right)$ and $S \in \mathfrak{G}_{s s}^{F}$, with $\mathfrak{L}=C_{\mathfrak{G}}(S)$. Then

$$
\sum_{N \in \mathfrak{L}_{\text {nil }}^{F}} f(S+N)=\mathcal{D} f(S) S t_{\mathfrak{G}}(S) .
$$

Proof. This follows immediately, taking $f_{1}=f$ and $f_{2}=1_{\mathfrak{G}}$ in (3.13).

We conclude this section with two analogues of the properties of the map corresponding to $\mathcal{D}$ in the group case, whose proof requires only the Mackey theorem (cf. [DM, §8]).

For any algebraic $\mathbb{F}_{q^{-}}$group $H$, write $\epsilon(H)$ for $(-1)^{r(H)}$, where $r(H)$ is the $\mathbb{F}_{q^{-}}$ rank of $H$.

\section{(3.15) Proposition.}

(i) Let $\mathfrak{G}, \mathfrak{P}=\mathfrak{L} \oplus \mathfrak{U}$ etc. be as above. Then $\mathcal{D}_{\mathfrak{G}} \circ \rho_{\mathfrak{L}}^{\mathfrak{G}}=\rho_{\mathfrak{L}}^{\mathfrak{G}} \circ \mathcal{D}_{\mathfrak{L}}$ and dually $\tau_{\mathfrak{L}}^{\mathfrak{G}} \circ \mathcal{D}_{\mathfrak{G}}=\mathcal{D}_{\mathfrak{L}} \circ \tau_{\mathfrak{L}}^{\mathfrak{G}}$.

(ii) Let $X \in \mathfrak{G}^{F}$ and write $\mathfrak{L}=C_{\mathfrak{G}}\left(X_{S}\right)$.

Then for any element $f \in \mathcal{C}\left(\mathfrak{G}^{F}\right)$, we have

$$
\mathcal{D}_{\mathfrak{G}} f(X)=\epsilon(G) \epsilon\left(C_{G}\left(X_{s}\right)^{\circ}\right)\left(\mathcal{D}_{\mathfrak{L}} \circ \operatorname{Res}_{\mathfrak{L}}^{\mathfrak{G}} f\right)(X) .
$$

The proofs of these statements are exactly the same as in the group case (see [DM, $\S \S 8.11,8.16])$.

\section{The Fourier transform}

Let $\psi: \mathbb{F}_{q}^{+} \longrightarrow \mathbb{C}^{\times}$be a non-trivial additive character of $\mathbb{F}_{q}$, fixed throughout this work.

Definition. The Fourier transform $\mathcal{F}_{\mathfrak{G}}=\mathcal{F}: \mathcal{C}\left(\mathfrak{G}^{F}\right) \longrightarrow \mathcal{C}\left(\mathfrak{G}^{F}\right)$ is defined by

$$
\begin{gathered}
\mathcal{F} f(X)=\left|\mathfrak{G}^{F}\right|^{-1 / 2} \sum_{Y \in \mathfrak{G}^{F}} \psi(\langle X, Y\rangle) f(Y) \\
\left(f \in \mathcal{C}\left(\mathfrak{G}^{F}\right), \quad X \in \mathfrak{G}^{F}\right)
\end{gathered}
$$

where $\langle$,$\rangle is the form on \mathfrak{G}$ postulated in (1.2).

The following properties of $\mathcal{F}$ all follow from simple calculations. 


\section{(4.2) Lemma.}

(i) $\mathcal{F}$ is an isometry of $\mathcal{C}\left(\mathfrak{G}^{F}\right)$ with respect to the form (, ) of (2.1).

(ii) $\mathcal{F}^{2} f=f^{-}$, where $f^{-}(X)=f(-X)\left(\right.$ for $\left.f \in \mathcal{C}\left(\mathfrak{G}^{F}\right), X \in \mathfrak{G}^{F}\right)$.

(iii) $\mathcal{F}^{4}=i d$.

(iv) $\mathcal{F}(f * g)=\mathcal{F} f \cdot \mathcal{F} g\left(\right.$ for $\left.f, g \in \mathcal{C}\left(\mathfrak{G}^{F}\right)\right)$.

(v) $\mathcal{F}(f \cdot g)=\mathcal{F} f * \mathcal{F} g\left(\right.$ for $\left.f, g \in \mathcal{C}\left(\mathfrak{G}^{F}\right)\right)$.

Proof. The proofs of (i), (ii) and (iii) are straightforward calculations.

(iv) Let $f, g \in \mathcal{C}\left(\mathfrak{G}^{F}\right)$ and $X \in \mathfrak{G}^{F}$. Then

$$
\begin{aligned}
\mathcal{F}(f * g)(X) & =\left|\mathfrak{G}^{F}\right|^{-1 / 2} \sum_{Y \in \mathfrak{G}^{F}} \psi(\langle X, Y\rangle) f * g(Y) \\
& =\left|\mathfrak{G}^{F}\right|^{-1 / 2} \sum_{Y \in \mathfrak{G}^{F}} \psi(\langle X, Y\rangle)\left|\mathfrak{G}^{F}\right|^{-1 / 2} \sum_{Z \in \mathfrak{G}^{F}} f(Y-Z) g(Z) \\
& =\left|\mathfrak{G}^{F}\right|^{-1} \sum_{Z \in \mathfrak{G}^{F}} \psi(\langle X, Z\rangle) g(Z) \sum_{Y \in \mathfrak{G}^{F}} \psi(\langle X, Y-Z\rangle) f(Y-Z) \\
& =\left|\mathfrak{G}^{F}\right|^{-1 / 2} \sum_{Z \in \mathfrak{G}^{F}} \psi(\langle X, Z\rangle) g(Z) \mathcal{F} f(X) \\
& =\mathcal{F} f(X) \cdot \mathcal{F} g(X), \text { as stated. }
\end{aligned}
$$

(v) Note first that for any pair $a, b \in \mathcal{C}\left(\mathfrak{G}^{F}\right)$, we have, by a simple application of (ii) above,

$$
\mathcal{F}^{2}(a * b)=\mathcal{F}^{2} a * \mathcal{F}^{2} b .
$$

Now apply $\mathcal{F}^{3}\left(=\mathcal{F}^{-1}\right)$ to both sides of (iv) above, obtaining

$$
\mathcal{F}^{4}(f * g)=f * g=\mathcal{F}^{3}(\mathcal{F} f \cdot \mathcal{F} g) .
$$

Applying $\mathcal{F}^{2}$ to both sides yields

$$
\begin{aligned}
\mathcal{F}^{2}(f * g) & =\mathcal{F}(\mathcal{F} f \cdot \mathcal{F} g) \\
& =\mathcal{F}^{2} f * \mathcal{F}^{2} g \quad(\text { by }(4.2 .1)) .
\end{aligned}
$$

Writing $a=\mathcal{F} f$ and $b=\mathcal{F} g$ in (4.2.2) gives $\mathcal{F}(a \cdot b)=\mathcal{F} a * \mathcal{F} b$.

(4.3) Lemma. Suppose $X$ is a semisimple element of $\mathfrak{G}$. Then the restriction of the form $\langle,\rangle_{\mathfrak{G}^{F}}(c f .(1.2))$ to $\mathcal{C}_{\mathfrak{G}}(X)$ is non-singular. If $X \in \mathfrak{G}^{F}$, it is defined over $\mathbb{F}_{q}$.

Proof. It follows from Borel [B, pp. 225 and 321] that $C_{\mathfrak{G}}(X)=$ Lie $\left(C_{G}(X)^{\circ}\right)$. If $T$ is a maximal torus of $C_{G}(X)^{\circ}$, it follows from (e.g.) [BT] that

$$
C_{\mathfrak{G}}(X)=\operatorname{Lie}(T) \oplus \sum_{\alpha \in \Phi_{X}} \mathfrak{G}_{\alpha}
$$

where $\Phi_{X}$ is the set of roots $\alpha$ of $G$ with respect to $T$ such that $d \alpha(X)=0$ and $\mathfrak{G}_{\alpha}$ is the root subspace of $\mathfrak{G}$ corresponding to $\alpha$.

Suppose $\beta$ is a root not in $\Phi_{X}$. By the $A d G$ invariance of $\langle,\rangle_{\mathfrak{G}}, \mathfrak{G}_{\beta}$ is orthogonal to $\operatorname{Lie}(T)$ and to each subspace $\mathfrak{G}_{\alpha}\left(\alpha \in \Phi_{X}\right)$. It follows that $\mathfrak{G}$ has an orthogonal direct decomposition. 


$$
\mathfrak{G}=C_{\mathfrak{G}}(X) \perp \sum_{\beta \in \Phi \backslash \Phi_{X}} \mathfrak{G}_{\beta}
$$

where $\Phi=\Phi(G, T)$ is the set of roots of $G$ with respect to $T$.

Hence any element $\xi \in C_{\mathfrak{G}}(X)$ in the radical of the restriction of $\langle$,$\rangle to C_{\mathfrak{G}}(X)$ is in the radical of $\langle,\rangle_{\mathfrak{G}}$, which by hypothesis is zero. Hence $\langle$,$\rangle is non-degenerate$ on $C_{\mathfrak{G}}(X)$. If $X \in \mathfrak{G}^{F}$, then $C_{\mathfrak{G}}(X)$ is F-stable and the restriction is defined over $\mathbb{F}_{q}$ since $\langle,\rangle \mathfrak{G}$ is.

If $L$ is any subgroup of $G$ of the form $L=C_{G}(X)^{\circ}$, where $X \in \mathfrak{G}$ is semisimple, then $L$ is connected and reductive, and we write $\langle,\rangle_{\mathfrak{L}}(\mathfrak{L}=$ Lie $(L))$ for the restriction of $\langle,\rangle_{\mathfrak{G}}$ to $\mathfrak{L}=C_{\mathfrak{G}}(X)$. If $X \in \mathfrak{G}^{F}$, then $\langle,\rangle_{\mathfrak{L}}$ satisfies the requirements of $(1.2)$ and hence we have a Fourier transform

$$
\mathcal{F}_{\mathfrak{L}}: \mathcal{C}\left(\mathfrak{L}^{F}\right) \longrightarrow \mathcal{C}\left(\mathfrak{L}^{F}\right)
$$

which enjoys all the properties of (4.2). Note that these remarks apply particularly to the case where $P=L U$ is an F-stable Levi decomposition of the F-stable parabolic subgroup $P$ of $G$.

(4.5) Theorem. Let $G$ be a connected reductive group over $\mathbb{F}_{q}$, with corresponding Frobenius endomorphism $F: G \longrightarrow G$. Let $P=L U$ be an F-stable Levi decomposition of the rational parabolic subgroup $P$ of $G$.

Let $\mathfrak{G}=$ Lie $(G), \mathfrak{L}=$ Lie $(L)$ etc., and let $\rho_{\mathfrak{L}}^{\mathfrak{G}}: \mathcal{C}\left(\mathfrak{L}^{F}\right) \longrightarrow \mathcal{C}\left(\mathfrak{G}^{F}\right)$ and $\tau_{\mathfrak{L}}^{\mathfrak{G}}:$ $\mathcal{C}\left(\mathfrak{G}^{F}\right) \longrightarrow \mathcal{C}\left(\mathfrak{L}^{F}\right)$ be the maps discussed in (3.3). Then with the above notation, we have

(i) $\tau_{\mathfrak{L}}^{\mathfrak{G}} \circ \mathcal{F}_{\mathfrak{G}}=\mathcal{F}_{\mathfrak{L}} \circ \tau_{\mathfrak{L}}^{\mathfrak{G}}$ and

(ii) $\mathcal{F}_{\mathfrak{G}} \circ \rho_{\mathfrak{L}}^{\mathfrak{G}}=\rho_{\mathfrak{L}}^{\mathfrak{G}} \circ \mathcal{F}_{\mathfrak{L}}$.

Proof.

(i) Let $f \in \mathcal{C}\left(\mathfrak{G}^{F}\right)$. Then for $X \in \mathfrak{L}^{F}$,

$$
\begin{aligned}
\tau_{\mathfrak{L}}^{\mathfrak{G}} \circ \mathcal{F}_{\mathfrak{G} f} f(X) & =\left|\mathfrak{U}^{F}\right|^{-1} \sum_{Y \in \mathfrak{U}^{F}} \mathcal{F}_{\mathfrak{G}} f(X+Y) \quad(\text { where } \mathfrak{U}=\operatorname{Lie}(U)) \\
& =\left|\mathfrak{U}^{F}\right|^{-1} \sum_{Y \in \mathfrak{U}^{F}}\left|\mathfrak{G}^{F}\right|^{-1 / 2} \sum_{Z \in \mathfrak{G}^{F}} \psi(\langle X+Y, Z\rangle) f(Z) \\
& =\left|\mathfrak{G}^{F}\right|^{-1 / 2}\left|\mathfrak{U}^{F}\right|^{-1} \sum_{Z \in \mathfrak{G}^{F}} \psi(\langle X, Z\rangle) f(Z) \sum_{Y \in \mathfrak{U}^{F}} \psi(\langle Y, Z\rangle) .
\end{aligned}
$$

But $\sum_{Y \in \mathfrak{U}^{F}} \psi(\langle Y, Z\rangle)=0$ unless $\langle Y, Z\rangle=0$ for all $Y \in \mathfrak{U}^{F}$ (since $Y \longmapsto$ $\psi(\langle Y, Z\rangle)$ is a character of the additive group of $\left.\mathfrak{U}^{F}\right)$, i.e. unless $Z \in\left(\mathfrak{U}^{F}\right)^{\perp}$ (the orthogonal subspace of $\left.\mathfrak{U}^{F}\right)$. But $\mathfrak{U}$ is the radical of $\langle$,$\rangle restricted to$ $\mathfrak{P}=\operatorname{Lie}(P)$ (cf. $[\mathrm{Sp} 1])$, whence by dimension, $\mathfrak{U}^{\perp}=\mathfrak{P}$, so that $\left(\mathfrak{U}_{F}^{\perp}\right)^{F}=\mathfrak{P}^{F}$. It follows that

$$
\sum_{Y \in \mathfrak{U}^{F}} \psi(\langle Y, Z\rangle)= \begin{cases}0 & \text { if } Z \notin \mathfrak{P}^{F} \\ \left|\mathfrak{U}^{F}\right| & \text { if } Z \in \mathfrak{P}^{F}\end{cases}
$$


Hence

$$
\begin{aligned}
\tau_{\mathfrak{L}}^{\mathfrak{G}} \circ \mathcal{F}_{\mathfrak{G}} f(X) & =\left|\mathfrak{G}^{F}\right|^{-1 / 2} \sum_{Z \in \mathfrak{P}^{F}} \psi(\langle X, Z\rangle) f(Z) \\
& =\left|\mathfrak{G}^{F}\right|^{-1 / 2} \sum_{Z \in \mathfrak{L}^{F}} \psi(\langle X, Z\rangle) \sum_{Y \in \mathfrak{U}^{F}} f(Z+Y) \\
& =\left|\mathfrak{G}^{F}\right|^{-1 / 2}\left|\mathfrak{U}^{F}\right| \sum_{Z \in \mathfrak{L}^{F}} \psi(\langle X, Z\rangle) \tau_{\mathfrak{L}}^{\mathfrak{G}} f(Z) \\
& =\mathcal{F}_{\mathfrak{L}} \circ \tau_{\mathfrak{L}}^{\mathfrak{G}} f(X)
\end{aligned}
$$

since $\left|\mathfrak{G}^{F}\right|=\left|\mathfrak{L}^{F}\right|\left|\mathfrak{U}^{F}\right|^{2}$.

(ii) This may be proved by a direct computation. Alternatively, one may use (i) as follows. First observe that repeated application of (i) gives

$$
\tau_{\mathfrak{L}}^{\mathfrak{G}} \circ \mathcal{F}_{\mathfrak{G}}^{i}=\mathcal{F}_{\mathfrak{L}}^{i} \circ \tau_{\mathfrak{L}}^{\mathfrak{G}} \quad \text { for } i=1,2,3, \cdots
$$

In particular, taking $i=3$ and using (4.2) (iii), it follows that (4.5.1) holds for $i=-1$ and hence for any $i \in \mathbb{Z}$.

Now take $f \in \mathcal{C}\left(\mathfrak{L}^{F}\right)$. To prove (ii), it will suffice to show that both sides of the equation, applied to $f$, have the same inner product with an arbitrary function $g \in \mathcal{C}\left(\mathfrak{G}^{F}\right)$ (by the non-degenerate nature of $\left.(,)_{\mathfrak{G} F}\right)$.

Let $g \in \mathcal{C}\left(\mathfrak{G}^{F}\right)$; then

$$
\begin{array}{rlr}
\left(\mathcal{F}_{\mathfrak{G}} \circ \rho_{\mathfrak{L}}^{\mathfrak{G}}(f), g\right)_{\mathfrak{G} F} & =\left(\rho_{\mathfrak{L}}^{\mathfrak{G}}(f), \mathcal{F}_{\mathfrak{G}}^{-1}(g)\right)_{\mathfrak{G} F} & \text { by }(4.2)(\mathrm{i}) \\
& =\left(f, \tau_{\mathfrak{L}}^{\mathfrak{G}} \circ \mathcal{F}_{\mathfrak{G}}^{-1}(g)\right)_{\mathfrak{L} F} & \text { by }(3.2) \\
& =\left(f, \mathcal{F}_{\mathfrak{L}}^{-1} \circ \tau_{\mathfrak{L}}^{\mathfrak{G}}(g)\right)_{\mathfrak{L} F} & \text { by }(4.5 .1) \\
& =\left(\mathcal{F}_{\mathfrak{L}} f, \tau_{\mathfrak{L}}^{\mathfrak{G}}(g)\right)_{\mathfrak{L} F} & \\
& =\left(\rho_{\mathfrak{L}}^{\mathfrak{G}} \circ \mathcal{F}_{\mathfrak{L}} f, g\right)_{\mathfrak{G} F} .
\end{array}
$$

Since $f \in \mathcal{C}\left(\mathfrak{L}^{F}\right)$ and $g \in \mathcal{C}\left(\mathfrak{G}^{F}\right)$ are arbitrary, the proof is complete.

It is now a simple matter to prove

(4.6) Theorem. With notation as in (4.5), the isometries $\mathcal{D}$ and $\mathcal{F}$ of $\mathcal{C}\left(\mathfrak{G}^{F}\right)$ commute.

Proof. We have in the notation of (3.4)

$$
\mathcal{F} \mathcal{D}=\sum_{\substack{P \supset B \\ F P=P}}(-1)^{n(P)} \mathcal{F} \circ \rho_{\mathfrak{L}_{P}}^{\mathfrak{G}} \circ \tau_{\mathfrak{L}_{p}}^{\mathfrak{G}} .
$$

But for any rational Levi subalgebra $\mathfrak{L}_{P}$, we have

$$
\begin{aligned}
\mathcal{F}_{\mathfrak{G}} \circ \rho_{\mathfrak{L}_{P}}^{\mathfrak{G}} \circ \tau_{\mathfrak{L}_{P}}^{\mathfrak{G}} & =\rho_{\mathfrak{L}_{P}}^{\mathfrak{G}} \circ \mathcal{F}_{\mathfrak{L}_{P}} \circ \tau_{\mathfrak{L}_{P}}^{\mathfrak{G}} & & \text { by }(4.5)(\mathrm{ii}) \\
& =\rho_{\mathfrak{L}_{P}}^{\mathfrak{G}} \circ \tau_{\mathfrak{L}_{P}}^{\mathfrak{G}} \circ \mathcal{F}_{\mathfrak{G}} & & \text { by }(4.5)(\mathrm{i}) .
\end{aligned}
$$

It follows that $\mathcal{F} \mathcal{D}=\mathcal{D} \mathcal{F}$. 


\section{Applications}

(5.1) Definition.

(i) For any element $X \in \mathfrak{G}^{F}$, define $\xi_{X} \in \mathcal{C}\left(\mathfrak{G}^{F}\right)$ by

$$
\xi_{X}(Y)= \begin{cases}1 & \text { if } Y \text { is in the } A d G^{F} \text {-orbit of } X, \\ 0 & \text { otherwise. }\end{cases}
$$

(ii) If $X \in \mathfrak{G}_{s s}^{F}$ (the set of semisimple elements of $\mathfrak{G}^{F}$ ), define $\eta_{X} \in \beta\left(\mathfrak{G}^{F}\right)$ by

$$
\eta_{X}(Y)= \begin{cases}1 & \text { if } Y_{s} \text { is in the } A d G^{F} \text {-orbit of } X \\ 0 & \text { otherwise }\end{cases}
$$

where $Y=Y_{s}+Y_{n}$ is the Jordan decomposition of $Y$.

With this notation, $\xi_{0}$ is the function supported on $\{0\} \subset \mathfrak{G}^{F}$, consistent with the notation of (2.5). Observe also that $\eta_{0}$ is the characteristic function of the nilpotent set $\mathfrak{G}_{n i l}^{F}$ in $\mathfrak{G}^{F}$.

(5.2) Proposition (Springer [Sp1]). Let $r=\operatorname{rank}(G)$ and $2 N=|\Phi|$ where $\Phi$ is the set of roots of $G$ with respect to the maximal torus $T$ ( $c f$. proof of (4.3)). Then

$$
\mathcal{F} S t_{\mathfrak{G}}=q^{r / 2} \eta_{0}
$$

Proof. We have (cf. (3.6)) $S t=\mathcal{D} 1_{\mathfrak{G}}$. Hence $\mathcal{F} S t_{\mathfrak{G}}=\mathcal{F} \mathcal{D}\left(1_{\mathfrak{G}}\right)=\mathcal{D} \mathcal{F}\left(1_{\mathfrak{G}}\right)$; moreover an easy calculation shows that $\mathcal{F} 1_{\mathfrak{G}}=\left|\mathfrak{G}^{F}\right|^{1 / 2} \xi_{0}$. Thus $\mathcal{F} S t_{\mathfrak{G}}=\left|\mathfrak{G}^{F}\right|^{1 / 2} \mathcal{D} \xi_{0}=$ $\left|\mathfrak{G}^{F}\right|^{1 / 2} \eta_{0} S t_{\mathfrak{G}}(0)^{-1}$ (by (3.10)). By (3.6), $S t_{\mathfrak{G}}(0)=q^{N}$, while $\left|\mathfrak{G}^{F}\right|=q^{2 N+r}$, which completes the proof.

(5.3) Corollary. We have, for any $X \in \mathfrak{G}^{F}, S t_{\mathfrak{G}}(X)=q^{-N} \sum_{Y \in \mathfrak{G}_{\text {nil }}^{F}} \psi(\langle X, Y\rangle)$.

Proof. Since $\mathcal{F} S t_{\mathfrak{G}}=q^{r / 2} \eta_{0}$, we have $\mathcal{F}^{2} S t_{\mathfrak{G}}=q^{r / 2} \mathcal{F} \eta_{0}$. But $\mathcal{F}^{2} S t_{\mathfrak{G}}=S t_{\mathfrak{G}}{ }^{-}=$ $S t_{\mathfrak{G}}$ by (3.6), and the stated formula follows.

(5.4) Corollary. Let $\mathcal{C}\left(\mathfrak{G}^{F}\right)_{\text {nil }}$ be the space of nilpotently supported functions in $\mathcal{C}\left(\mathfrak{G}^{F}\right)$. We have $\mathcal{F}\left(\mathcal{C}\left(\mathfrak{G}^{F}\right)_{\text {nil }}\right)=S t_{\mathfrak{G}} * \mathcal{C}\left(\mathfrak{G}^{F}\right)$.

Proof. Clearly $\mathcal{C}\left(\mathfrak{G}^{F}\right)_{\text {nil }}=\eta_{0} \cdot \mathcal{C}\left(\mathfrak{G}^{F}\right)$. But for $f \in \mathcal{C}\left(\mathfrak{G}^{F}\right), \mathcal{F}\left(\eta_{0} \cdot f\right)=\mathcal{F} \eta_{0} * \mathcal{F} f=$ $q^{-r / 2} S t_{\mathfrak{G}} * \mathcal{F} f$. Since $f$ is arbitrary, the result follows.

(5.5) Corollary. We have $\mathcal{F}\left(\mathcal{C}\left(\mathfrak{G}^{F}\right)_{s s}\right)=\eta_{0} * \mathcal{C}\left(\mathfrak{G}^{F}\right)$.

Proof. Any function in $\mathcal{C}\left(\mathfrak{G}^{F}\right)_{s s}$ is of the form $f=S t_{\mathfrak{G}} \cdot g$ for some $g \in \mathcal{C}\left(\mathfrak{G}^{F}\right)$. One now argues as in (5.4).

The following result is a sharper version of the last two statements.

(5.6) Theorem. Let $G$ etc. be as above and let $\mathcal{D}$ be the "duality" map of (3.4).

With the above notation, we have, for any $f \in \mathcal{C}\left(\mathfrak{G}^{F}\right)$

(i) $\mathcal{D}\left(\eta_{0} \cdot f\right)=\eta_{0} \cdot \mathcal{D} f$,

(ii) $\mathcal{D}\left(S t_{\mathfrak{G}} * f\right)=S t_{\mathfrak{G}} * \mathcal{D} f$.

Proof.

(i) Take $b=\eta_{0}$ in Theorem (3.10). The result follows immediately.

(ii) Apply $\mathcal{F}$ to the equation (i) above: 


$$
\mathcal{F}\left(\mathcal{D}\left(\eta_{0} \cdot f\right)\right)=\mathcal{F}\left(\eta_{0} \cdot \mathcal{D} f\right)=q^{-r / 2} S t_{\mathfrak{G}} * \mathcal{F} \mathcal{D} f \quad \text { by }(5.2) .
$$

Using Theorem (4.6), we obtain

$$
\mathcal{D}\left(\mathcal{F} \eta_{0} * \mathcal{F} f\right)=\mathcal{D}\left(q^{-r / 2} S t_{\mathfrak{G}} * \mathcal{F} f\right)=q^{-r / 2} S t_{\mathfrak{G}} * \mathcal{D} \mathcal{F} f
$$

Dividing by $q^{-r / 2}$ and replacing $\mathcal{F} f$ by $f$ gives the stated result.

The next result generalises (5.2).

(5.7) Theorem. For any semisimple element $S \in \mathfrak{G}^{F}$, write $\pi_{S}$ for the orthogonal projection from $\mathfrak{G}^{F}$ to $C_{\mathfrak{G}}(S)^{F}$ (cf. (4.3.2)). Then with notation as in (5.1) we have

$$
\mathcal{F} \eta_{S}(X)=q^{N(S)} S t_{C_{\mathfrak{G}}(S)}\left(\pi_{S}(X)\right) \mathcal{F} \xi_{S}(X)
$$

where $N(S)$ is the number of positive roots of $C_{G}(S)^{\circ}$ (with respect to a maximal torus).

Proof. We have

$$
\begin{aligned}
\mathcal{F} \eta_{S}(X) & =\left|\mathfrak{G}^{F}\right|^{-1 / 2} \sum_{Y \in \mathfrak{G}^{F}} \psi(\langle X, Y\rangle) \eta_{S}(Y) \\
& =\left|\mathfrak{G}^{F}\right|^{-1 / 2} \sum_{Y \in \mathcal{O}_{S}} \sum_{Z \in C_{\mathfrak{G}}(Y)_{\text {nil }}^{F}} \psi(\langle X, Y+Z\rangle
\end{aligned}
$$

(in the notation of the proof of (3.13))

$$
=\left|\mathfrak{G}^{F}\right|^{-1 / 2} \sum_{Y \in \mathcal{O}_{S}} \sum_{Z \in C_{\mathfrak{G}}(Y)_{\text {nil }}^{F}} \psi(\langle X, Y\rangle) \psi(\langle X, Z\rangle) .
$$

But by (5.3), we have for any $Y \in \mathcal{O}_{S}$

$$
\sum_{Z \in C_{\mathfrak{G}}(Y)_{n i l}^{F}} \psi(\langle X, Z\rangle)=q^{N(S)} S t_{C_{\mathfrak{G}}(Y)}\left(\pi_{Y}(X)\right)
$$

Moreover if $Y=\operatorname{Adg} S\left(g \in G^{F}\right)$, since $\langle$,$\rangle is A d G$-invariant, we have $\pi_{Y}(X)=$ $A d g \pi_{S}(X)$ and $S t_{C_{\mathfrak{G}}(Y)}=S t_{C_{\mathfrak{G}}(S)} \circ A d\left(g^{-1}\right)$. It follows that for each $Y \in \mathcal{O}_{S}$, $S t_{C_{\mathfrak{G}}(Y)}\left(\pi_{Y}(X)\right)=S t_{C_{\mathfrak{G}}(S)}\left(\pi_{S}(X)\right)$.

Substituting into the above expression for $\mathcal{F} \eta_{S}(X)$, we obtain

$$
\begin{aligned}
\mathcal{F} \eta_{S}(X) & =\left|\mathfrak{G}^{F}\right|^{-1 / 2} q^{N(S)} S t_{C_{\mathfrak{G}}(S)}\left(\pi_{S}(X)\right) \sum_{Y \in \mathcal{O}_{S}} \psi(\langle X, Y\rangle) \\
& =q^{N(S)} S t_{C_{\mathfrak{E}}(S)}\left(\pi_{S}(X)\right) \mathcal{F} \xi_{S}(X) .
\end{aligned}
$$


(5.8) Corollary. In the notation of (5.8), we have $\mathcal{F} \eta_{S}(X)=0$ unless $\pi_{S}(X)$ is semisimple.

As a further application of these ideas, observe that the result $[\mathrm{Lu} 2,(8.5)]$ of Lusztig (which generalises (4.2.1) of $[\mathrm{K}]$ ) asserts that for certain $f \in \mathcal{C}\left(\mathfrak{G}^{F}\right)_{n i l}$, one has

$$
\mathcal{D} f=c(f) \mathcal{F} f \cdot \eta_{0}
$$

where $c(f)$ is a constant depending on $f$.

The following statement is a simple consequence of (4.6) and (5.6).

$$
\begin{aligned}
& \text { A function } f \in \mathcal{C}\left(\mathfrak{G}^{F}\right) \text { satisfies the condition of (5.9) if and only if } \\
& \mathcal{F} f=q^{-r / 2} c(f)^{-1} \mathcal{D} f * S t_{\mathfrak{G}}
\end{aligned}
$$

\section{Application to Green functions}

We assume for simplicity in this section that $G$ is F-split and that $C_{G}(X)$ is connected for $X \in \mathfrak{G}_{s s}$. Recall $[\mathrm{L} 1, \S 5]$ that we have a "rational classification" of the semisimple orbits of $\mathfrak{G}^{F}$, which associates to the orbit of $X \in \mathfrak{G}_{s s}^{F}$ the conjugacy class $\omega(X)$ in $W$ which corresponds to the $G^{F}$-class of maximally split tori of $C_{G}(X)$.

Let $S_{w}$ be a regular semisimple element of $\mathfrak{G}^{F}$ such that $\omega\left(S_{w}\right)=(w)$ (for $w \in W$, we write $(w)$ for the $W$-conjugacy class of $w \in W$ ). Then $\xi_{S_{w}}$ is (cf. (5.1) (i)) the characteristic function of the $G^{F}$-orbit of $S_{w}$. Springer [Sp2] has studied (geometrically) the functions $\mathcal{F} \xi_{S_{w}}$ and proved in particular that

(i) The function $\mathcal{F} \xi_{S_{w}} \cdot \eta_{0}$ depends only on $(w)$ (and not on $S_{w}$ ) (recall that $\eta_{0}$ is the characteristic function of $\left.\mathfrak{G}_{n i l}^{F}\right)$.

(ii) The function $Q_{w}=\epsilon(w) q^{r / 2} \mathcal{F} \xi_{S_{w}} \cdot \eta_{0}$ is the "Green function" of $G^{F}$ (defined on $\left.\mathfrak{G}_{\text {nil }}^{F}\right)$ corresponding to the $G^{F}$-class of rational tori of $G$ parametrized by the class $(w) \subset W$. (Here $\epsilon(w)$ is the alternating character $\epsilon$ of $W$ evaluated at $w \in W$.)

As a simple consequence of our main theorem, we have (cf. [K2, (3.2.9)])

(6.2) Proposition. We have, in the notation of (6.1), $\mathcal{D} Q_{w}=\epsilon(w) Q_{w}$.

Proof. Note first that since $S_{w}$ is regular semisimple, $\xi_{S_{w}} \in \mathcal{B}\left(\mathfrak{G}^{F}\right) \cap \mathcal{C}\left(\mathfrak{G}^{F}\right)_{s s}$. Hence by (3.11), we have

$$
\mathcal{D} \xi_{S_{w}}=S t_{\mathfrak{G}}\left(S_{w}\right) \xi_{S_{w}}
$$

Moreover by (3.6), $S t_{\mathfrak{G}}\left(S_{w}\right)=(-1)^{r(G)-r(T)}$ where $T$ is the (unique) maximal torus such that $S_{w} \in$ Lie $T$. But (see, e.g. $\left.[\mathrm{L},(5.5)]\right)(-1)^{r(G)-r(T)}=\epsilon(w)$, whence

$$
\mathcal{D} \xi_{S_{w}}=\epsilon(w) \xi_{S_{w}} .
$$


It follows that

$$
\begin{array}{rlr}
\mathcal{D} Q_{w} & =\epsilon(w) q^{r / 2} \mathcal{D}\left(\mathcal{F} \xi_{S_{w}} \cdot \eta_{0}\right) & \\
& =\epsilon(w) q^{r / 2} \mathcal{D}\left(\mathcal{F} \xi_{S_{w}}\right) \cdot \eta_{0} & \text { by }(5.6)(\mathrm{i}) \\
& =\epsilon(w) q^{r / 2} \mathcal{F}\left(\mathcal{D} \xi_{S_{w}}\right) \cdot \eta_{0} & \text { by }(4.6) \\
& =\epsilon(w) q^{r / 2} \mathcal{F}\left(\epsilon(w) \xi_{S_{w}}\right) \cdot \eta_{0} & \text { by }(6.2 .2) \\
& =\epsilon(w) Q_{w} . &
\end{array}
$$

(6.3) Definition. For $X \in \mathfrak{G}$, define the variety $\mathcal{B}_{X}=\left\{B^{\prime} \in \mathcal{B} \mid X \in\right.$ Lie $\left.B^{\prime}\right\}$, where $\mathcal{B}(\cong G / B)$ is the flag variety of $G$ (i.e. the variety of Borel subgroups of $G)$.

It is known that the $\ell$-adic cohomology of $B_{X}$ vanishes in odd dimension (for $\ell$ a prime not dividing $q$ ). Moreover Springer [Sp2] has shown there is an action of $W$ on the cohomology $H^{*}\left(\mathcal{B}_{X}, \bar{Q}_{\ell}\right)$ such that we have

(6.4) Proposition. Let $X \in \mathfrak{G}_{\text {nil }}^{F}$. Then the values of $Q_{w}$ are given by

$$
Q_{w}(X)=\sum_{i \geq 0} \operatorname{tr}\left(F w, H^{2 i}\left(\mathcal{B}_{X}, \overline{\mathbb{Q}}_{\ell}\right)\right)
$$

This statement is Theorem (4.4) of [Sp2] (see also [Sh, (13.2.1)]).

(6.5) Definition. Define $\widetilde{Q}_{w} \in \mathcal{C}\left(\mathfrak{G}^{F}\right)$ by $\widetilde{Q}_{w}(X)=\sum_{i \geq 0} \operatorname{tr}\left(F w, H^{2 i}\left(\mathcal{B}_{X}, \overline{\mathbb{Q}}_{\ell}\right)\right)$ $\left(\right.$ any $\left.X \in \mathfrak{G}^{F}\right)$.

Observe that $Q_{w}=\widetilde{Q}_{w} \cdot \eta_{0}\left(\eta_{0}\right.$ as in (5.1) (ii)).

The functions $\widetilde{Q}_{\chi}=|W|^{-1} \sum_{W \in W} \chi(w) \widetilde{Q}_{w}$ (for $\chi$ an irreducible character of $W$ ) may be interpreted as the characteristic functions of certain admissible complexes of sheaves on $\mathfrak{G}$ (see [Sh, $\S \S 13,14]$ ). There is a notion of Fourier transform for

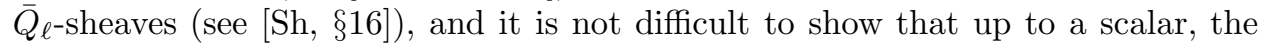
characteristic function of a Fourier transform is the Fourier transform of the original characteristic function ([Sh, (16.2.1)]).

These considerations lead to the following result, which is essentially due to Brylinski and Kashiwara (cf. [Sh, (17.7)]).

(6.6) Proposition. With the above notation, we have, for $\chi$ an irreducible character of $W$,

(i) $\mathcal{F} \widetilde{Q}_{\chi}=\widetilde{Q}_{\epsilon \chi} \cdot \eta_{0}$.

(ii) For $w \in W$, we have

$$
\mathcal{F} \widetilde{Q}_{w}=\epsilon(w) q^{r / 2} Q_{w}
$$

The statement (i) is a consequence of (17.7) and (16.2.1) of [Sh], while (ii) follows from (i) in a formal way.

(6.7) Theorem. We have, with the above notation, for $w \in W$

$$
\widetilde{Q}_{w}=q^{r / 2} \xi_{S_{w}} * S t_{\mathfrak{G}}
$$

Proof. We have (from (6.1))

$$
Q_{w}=\epsilon(w) q^{r / 2} \mathcal{F} \xi_{S_{w}} \cdot \eta_{0} .
$$


Taking Fourier transforms (and using (5.2)), we obtain

$$
\mathcal{F} Q_{w}=\epsilon(w) \xi_{S_{w}} * S t_{\mathfrak{G}} .
$$

But from (6.6) (ii), we have

$$
\mathcal{F} Q_{w}=q^{r / 2} \epsilon(w) \widetilde{Q}_{w}
$$

(since clearly $\left.\widetilde{Q}_{w}(X)=\widetilde{Q}_{w}(-X)\right)$.

Comparing (6.7.1) and (6.7.2) completes the proof.

(6.8) Corollary. Let $\widetilde{Q}_{w} \in \mathcal{C}\left(\mathfrak{G}^{F}\right)$ be given by $\widetilde{Q}_{w}(X)=\sum_{i} \operatorname{tr}\left(w F, H^{2 i}\left(\mathcal{B}_{X}, \overline{\mathbb{Q}}_{\ell}\right)\right)$ $\left(\right.$ any $\left.X \in \mathfrak{G}^{F}\right)$. Then $\mathcal{D} \widetilde{Q}_{w}=\epsilon(w) \widetilde{Q}_{w}$.

Proof. By (6.7), $\widetilde{Q}_{w}$ is a constant multiple of $\xi_{S_{w}} * S t_{\mathfrak{G}}$. But by (5.6) (ii) we have $\mathcal{D}\left(\xi_{S_{w}} * S t_{\mathfrak{G}}\right)=\mathcal{D} \xi_{S_{w}} * S t_{\mathfrak{G}}$ and we have seen above (6.2.1) that $\mathcal{D} \xi_{S_{w}}=\epsilon(w) \xi_{S_{w}}$. The result follows.

(6.9) Corollary. For any $X \in \mathfrak{G}^{F}, \widetilde{Q}_{w}(X)$ is a rational integer.

\section{Geometric consequences}

In this section we interpret the results of $\S 5$ in terms of the cohomology of certain subvarieties of the variety $\mathcal{T}$ of maximal tori of $G$. In order to do this, we give expressions for $S t_{\mathfrak{G}}$ and for $\eta_{0}$ (the characteristic function of $\mathfrak{G}_{n i l}^{F}$ ) in terms of the set of rational maximal tori of $G$. Let $T_{0}$ be a maximally split maximal torus of $G$. Write $N_{0}=N_{G}\left(T_{0}\right)$. Then $N_{0} / T_{0} \cong W=W\left(G, T_{0}\right)$, the Weyl group of $G$ with respect to $T_{0}$.

\section{(7.1) Definition.}

(i) Write $\mathcal{T}$ for the variety of maximal tori of $G$. Thus $\mathcal{T}=G / N_{0}$.

(ii) Denote by $\pi: G / T_{0} \longrightarrow \mathcal{T}$ the projection given by $\pi\left(g T_{0}\right)=g T_{0} g^{-1}={ }^{g} T_{0}$.

Then $\pi$ is an unramified covering map with fibre $W$.

The $G^{F}$-orbits on $\mathcal{T}^{F}$ are in canonical bijection with the F-conjugacy classes of $W$ (cf. [L1]). Hence for each $T \in \mathcal{T}^{F}$, we may speak of $\omega(T)$, the corresponding F-conjugacy class of $W$. If $\chi$ is an F-class function on $W$, we write

$$
\chi(T)=: \chi(\omega(T)) \quad\left(T \in T^{F}\right) .
$$

In particular, this definition applies when $\chi=\epsilon$, the alternating character of $W$.

(7.3) Proposition. We have, for $X \in \mathfrak{G}^{F}, S t_{\mathfrak{G}}(X)=\sum_{\substack{T \in \mathcal{T}^{F} \\ \text { Lie } T \ni X}} \epsilon(T)$.

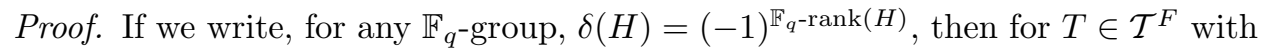
$\omega(T)=(w)$, we have $\delta(T)=\delta(G) \epsilon(w)$ (see, e.g. [L1, (5.5)]). Hence

$$
\sum_{T \in \mathcal{T}^{F}} \delta(T)=\delta(G) \sum_{T \in \mathcal{T}^{F}} \epsilon(T)=\delta(G) q^{N} \quad \text { by }[\mathrm{L} 1(1.12)] .
$$


Now for $X \in \mathfrak{G}^{F},\{T \in \mathcal{T} \mid$ Lie $T \ni X\}=\left\{T \in \mathcal{T} \mid T \subset C_{G}(X)^{\circ}\right\}$ (see [B, pp. $225,321])$. It follows that

$$
\sum_{\substack{T \in \mathcal{T}^{F} \\ \text { Lie } T \ni X}} \delta(T)=\delta\left(C_{G}(X)^{\circ}\right) q^{N(X)} .
$$

Using the relation $\delta(T)=\delta(G) \epsilon(T)$, we deduce that

$$
\sum_{\substack{T e \mathcal{T} F \\ \text { LieT } T X}} \epsilon(T)=\delta(G) \delta\left(C_{G}(X)^{\circ}\right) q^{N(X)} .
$$

Comparing with (3.6) completes the proof.

(7.4) Definition. Let $\chi$ be any F-class function on $W$ (i.e. $\chi\left(w_{1}^{-1} w F\left(w_{1}\right)\right)=\chi(w)$ for all $\left.w, w_{1} \in W\right)$. Define $\sigma_{\chi} \in \mathcal{C}\left(\mathfrak{G}^{F}\right)$ by

$$
\sigma_{\chi}(X)=\sum_{\substack{T \in \mathcal{T} F \\ \text { Lie } T \ni X}} \chi(T) .
$$

Then (7.3) may be expressed by $\sigma_{\epsilon}=S t_{\mathfrak{G}}$. Note that all the functions $\sigma_{\chi}$ are supported on $\mathfrak{G}_{s s}^{F}$.

The following result is not essential to the main subject of this section. It may be proved using the methods of $[\mathrm{L} 1, \S 1]$.

(7.5) Theorem. With notation as in (7.4), we have

$$
\sigma_{\chi}=|W|^{-1} \sum_{w \in W} \chi(w) \operatorname{Ind} d_{\mathfrak{T}_{w}}^{\mathfrak{G}}(1)
$$

where $\mathfrak{T}_{w}=$ Lie $T_{w}$ and $T_{w} \in \mathcal{T}^{F}$ is of type $(w)$.

This immediately implies the Lie-algebraic analogue of the result of Srinivasan $[\mathrm{Sr}]$.

(7.6) Corollary. We have $S t_{\mathfrak{G}}=|W|^{-1} \sum_{w \in W} \epsilon(w) \operatorname{Ind}_{\mathfrak{T}_{w}}^{\mathfrak{G}}(1)$.

(7.7) Proposition. In the notation of (7.4), we have, for $X \in \mathfrak{G}^{F}$

$$
\mathcal{F} \sigma_{\chi}(X)=q^{r / 2-N} \sum_{T \in \mathcal{T}_{X}^{F}} \chi(T)
$$

where $\mathcal{T}_{X}=\{T \in \mathcal{T} \mid\langle$ Lie $T, X\rangle=0\}$.

Proof.

$$
\begin{aligned}
\mathcal{F} \sigma_{\chi}(X) & =\left|\mathfrak{G}^{F}\right|^{-1 / 2} \sum_{Y \in \mathfrak{G}^{F}} \psi(\langle X, Y\rangle) \sigma_{\chi}(Y) \\
& =\left|\mathfrak{G}^{F}\right|^{-1 / 2} \sum_{Y \in \mathfrak{G}^{F}} \psi(\langle X, Y\rangle) \sum_{\substack{T \in \mathcal{T}_{F}^{F} \\
\text { Lie } T \ni Y}} \chi(T) \\
& =\left|\mathfrak{G}^{F}\right|^{-1 / 2} \sum_{T \in \mathcal{T}^{F}} \chi(T) \sum_{Y \in \operatorname{Lie} T} \chi(\langle X, Y\rangle) .
\end{aligned}
$$


But the inner sum is zero unless $T \in \mathcal{T}_{X}$, in which case it is equal to $\left|(\operatorname{Lie} T)^{F}\right|=$ $q^{r}$. Hence

$$
\begin{aligned}
\mathcal{F} \sigma_{\chi}(X) & =\left|\mathfrak{G}^{F}\right|^{-1 / 2} q^{r} \sum_{T \in \mathcal{T}_{X}^{F}} \chi(T) \\
& =q^{r / 2-N} \sum_{T \in \mathcal{T}_{X}^{F}} \chi(T) \quad \text { as asserted. }
\end{aligned}
$$

Applying (7.7) to the case $\chi=\epsilon$, we see

(7.8) Corollary. We have, for $X \in \mathfrak{G}^{F}$,

$$
\mathcal{F} S t(X)=q^{r / 2-N} \sum_{T \in \mathcal{T}_{X}^{F}} \epsilon(T) .
$$

Combining (7.8) and (5.2), we obtain

(7.9) Theorem. Let $X \in \mathfrak{G}^{F}$. Then

$$
\sum_{T \in \mathfrak{T}_{X}^{F}} \epsilon(T)= \begin{cases}0 & \text { unless } X \in \mathfrak{G}_{\text {nil }}^{F} \\ q^{N} & \text { if } X \in \mathfrak{G}_{\text {nil }}^{F} .\end{cases}
$$

We show finally that (7.3) and (7.9) have a geometric interpretation which points to a generalisation to real Lie groups. For this we recall the following result of Deligne [D, (1.1.1) p. 169].

(7.10). Let $X$ be a separated scheme of finite type over $\mathbb{F}_{q}$ and let $\mathcal{S}$ be a $\overline{\mathbb{Q}}_{\ell}$-sheaf on $X$, stable under the Frobenius map $F: X \longrightarrow X$. Then

$$
\sum_{x \in X^{F}} \operatorname{tr}\left(F, \mathcal{S}_{x}\right)=\sum_{i}(-1)^{i} \operatorname{tr}\left(F, H_{c}^{i}(X, \mathcal{S})\right)
$$

where $\mathcal{S}_{x}$ is the stalk of $\mathcal{S}$ at $x$.

Now consider the sheaf $\pi_{*} \overline{\mathbb{Q}}_{\ell}=\mathcal{S}$ on $\mathcal{T}$ (see (7.1) (ii)), where $\overline{\mathbb{Q}}_{\ell}$ is the constant sheaf on $G / T_{0}$. This is a local system on $T$ corresponding to the regular representation of $W$.

(7.11) Definition. For any irreducible character $\chi$ of $W$, let $\mathcal{S}_{\chi}$ be an irreducible summand of $\mathcal{S}$ which corresponds to $\chi$.

Thus $\mathcal{S}_{\chi}$ is a local system on $\mathcal{T}$.

(7.12) Lemma. Let $\epsilon$ be the alternating character of $W$. Then the local system $\mathcal{S}_{\epsilon}$ is F-stable and for any $T \in \mathcal{T}^{F}$, we have

$$
\operatorname{tr}\left(F, \mathcal{S}_{\epsilon, T}\right)=\epsilon(T)
$$

Proof. $\mathcal{S}_{\epsilon}$ is F-stable because $\epsilon$ is F-invariant and the $\epsilon$ - isotypic component of the regular representation of $W$ is canonical.

For the second part, observe that if $T=g T_{0} g^{-1}, \pi^{-1}(T)=\left\{g w T_{0} \mid w \in W\right\}$ and so $\mathcal{S}_{\epsilon, T}$ may be taken as $\overline{\mathbb{Q}}_{\ell} \sum_{w \in W} \epsilon(w) g w T_{0}$. 
But $F\left(\sum_{w \in W} \epsilon(w) g w T_{0}\right)=\sum_{w \in W} \epsilon(w) F(g) F(w) T_{0}$.

Moreover $g^{-1} F(g)=w(T) \in W$, so that

$$
\begin{aligned}
F\left(\sum_{w \in W} \epsilon(w) g w T_{0}\right) & =\sum_{w \in W} \epsilon(w) g w(T) F(w) T_{0} \\
& =\epsilon(w(T)) \sum_{w \in W} \epsilon(w(T) F(w)) g w(T) F(w) T_{0} \\
& =\epsilon(w(T)) \sum_{w \in W} \epsilon(w) g w T_{0} \\
& =\epsilon(T) \sum_{w \in W} \epsilon(w) g w T_{0} .
\end{aligned}
$$

We may now give geometric interpretations of (7.3) and (7.9).

(7.13) Theorem. Let $\mathcal{S}_{\epsilon}$ be the local system on the variety $\mathcal{T}$ of maximal tori of $G$ described above. For $X \in \mathfrak{G}^{F}$ let

$$
\begin{aligned}
\mathcal{T}^{X} & =\{T \in \mathcal{T} \mid \text { Lie } T \ni X\} \quad \text { and } \\
\mathcal{T}_{X} & =\{T \in \mathcal{T} \mid\langle\text { Lie } \mathfrak{T}, X\rangle=0\} .
\end{aligned}
$$

Then we have

(i) $\sum(-1)^{i} \operatorname{tr}\left(F, H_{c}^{i}\left(\mathcal{T}^{X}, \mathcal{S}_{\epsilon}\right)\right)=S t_{\mathfrak{G}}(X)$

(ii) $\sum_{i}(-1)^{i} \operatorname{tr}\left(F, H_{c}^{i}\left(\mathcal{T}_{X}, \mathcal{S}_{\epsilon}\right)\right)= \begin{cases}0 & \text { unless } X \text { is nilpotent } \\ q^{N} & \text { if } X \text { is nilpotent. }\end{cases}$

Proof. This statement is just a combination of the results (7.3) and (7.9) taking account of the fixed point theorem (7.10). The trace of $F$ on the stalks of $\mathcal{S}_{\epsilon}$ is given by (7.12).

We observe finally that the local systems $\mathcal{S}_{\chi}(\chi \in \hat{W})$ exist in any characteristic. The result (7.13) therefore opens the possibility of studying a "Steinberg function" for (e.g.) a real Lie group.

\section{REFERENCES}

[B] A. Borel, Linear algebraic groups, Benjamin, N.Y. (1969). MR 40:4273

[BT] A. Borel and J. Tits, Groupes réductifs, Publ. Math IHES 27 (1965), 55-152. MR 34:7527

[C] C.W. Curtis, Truncation and duality in the character ring of a finite group of Lie type, J. of Algebra 62 (1980), 320-332. MR 81e:20011

[CLT] C.W. Curtis, G.I. Lehrer and J. Tits, Spherical buildings and the character of the Steinberg representation, Inventiones Math. 58 (1980), 201-210. MR 81f:20060

[D] P. Deligne, Cohomologie Étale (SGA 4 $\frac{1}{2}$ ), Springer L.N.M., Springer-Verlag Berlin 509 (1977). MR 57:3132

[DL] P. Deligne and G. Lusztig, Representations of reductive groups over finite fields, Ann. Math. 103 (1976), 103-161. MR 52:14076

[DM] F. Digne and J. Michel, Representations of finite groups of Lie type, Cambridge U.P., Cambridge (1991). MR 92g:20063

[HL] R.B. Howlett and G.I. Lehrer, On Harish-Chandra induction and restriction for modules of Levi subgroups, J. of Algebra 165 (1994), 172-183. MR 95d:20025

[K] N. Kawanaka, Fourier transforms of nilpotently supported invariant functions on a simple Lie algebra over a finite field, Inventiones Math. 69 (1982), 411-435. MR 84c:20053 
[K2] N. Kawanaka, Generalized Gelfand-Graev representations and Ennola duality, Adv. St. Pure Math. 6 (1985), 175-206. MR 87e:20075

[L1] G.I. Lehrer, Rational tori, semisimple orbits and the topology of hyperplane complements, Commentarii Math. Helv. 67 (1992), 226-251. MR 93e:20065

[L2] G.I. Lehrer, Characters and the Jordan decomposition in reductive groups over finite fields, U. Warwick preprint series (1980).

[Lu1] G. Lusztig, A unipotent support for irreducible representations, Advances in Math. 94 (1992), 139-179. MR 94a:20073

[Lu2] G. Lusztig, Fourier transforms on a semisimple Lie algebra over $\mathbb{F}_{q}$, in "Algebraic groups Utrecht 1986", Springer L.N.M. 12 (1987), 177-188. MR 89b:17015

[MS] J.G.M. Mars and T.A. Springer, Character sheaves, Soc. Math. France Astérisque 173174 (1989), 111-198. MR 91a:20044

[Sh] T. Shoji, Geometry of orbits and Springer correspondence, Soc. Math. France Astérisque 168 (1988), 61-140. MR 91b:20057

[Sp1] T.A. Springer, The Steinberg function of a finite Lie algebra, Inventiones Math. $\mathbf{5 8}$ (1980), 211-215. MR 81g:20090

[Sp2] T.A. Springer, Trigonometric sums, Green functions of finite groups and representations of Weyl Groups, Inventiones Math. 36 (1976), 173-207. MR 56:491

[Sp2] T.A. Springer, The unipotent variety of a semisimple group, Proc. Coll. Alg. Geom., Tata Institute (1969), 373-391. MR 41:8429

[Sr] B. Srinivasan, On the Steinberg character of a finite simple group of Lie type, J. Aust. Math. Soc. 12 (1977), 1-14. MR 45:411

School of Mathematics and Statistics, University of Sydney, Sydney N.S.W. 2006, Australia 